PROCEEDINGS OF THE

AMERICAN MATHEMATICAL SOCIETY

Volume 140, Number 11, November 2012, Pages 4011-4016

S 0002-9939(2012)11253-5

Article electronically published on February 29, 2012

\title{
GENERATING THE MÖBIUS GROUP WITH INVOLUTION CONJUGACY CLASSES
}

\author{
ARA BASMAJIAN AND KARAN PURI \\ (Communicated by Michael Wolf)
}

\begin{abstract}
A $k$-involution is an involution with a fixed point set of codimension $k$. The conjugacy class of such an involution, denoted $S_{k}$, generates $\operatorname{Möb}(n)$ (the group of isometries of hyperbolic $n$-space) if $k$ is odd and its orientation-preserving subgroup if $k$ is even. In this paper, we supply effective lower and upper bounds for the $S_{k}$ word length of $\operatorname{Möb}(n)$ if $k$ is odd and the $S_{k}$ word length of $\mathrm{Möb}^{+}(n)$ if $k$ is even. As a consequence, for a fixed codimension $k$, the length of $\mathrm{Möb}^{+}(n)$ with respect to $S_{k}, k$ even, grows linearly with $n$, with the same statement holding for $\operatorname{Möb}(n)$ in the odd case. Moreover, the percentage of involution conjugacy classes for which $\mathrm{Möb}^{+}(n)$ has length two approaches zero as $n$ approaches infinity.
\end{abstract}

\section{INTRODUCTION AND RESULTS}

Let $G$ be a group and $S$ a set of symmetric generators for a supergroup of $G$; $S$ is not necessarily a subset of $G$, but every element can be written as a product of elements from $S$. For $g \in G$, the length of $g$ with respect to $S$ (or $S$-length) is the minimal number of elements of $S$ needed to express $g$ as their product. The supremum over all group element lengths is called the length of $G$ with respect to $S$ (or simply the $S$-length of $G$ ), and is denoted by $|G|$.

We are interested in the set $S_{k} \subset \operatorname{Möb}(n)$ of involutions with a codimension $k$ fixed point set acting on hyperbolic space, $\mathbb{H}^{n}$.

Theorem 1.1. Let $n \geq 2$ and $k=1,2, \ldots, n-1$.

- If $k$ is even, $S_{k}$ generates $M \ddot{o} b^{+}(n)$ and satisfies

$$
\frac{n(n+1)}{2 k(n-k+1)} \leq\left|M \ddot{o} b^{+}(n)\right|_{k} \leq 2 n+4 .
$$

- If $k$ is odd, $S_{k}$ generates $M o ̈ b(n)$ and satisfies

$$
\frac{n(n+1)}{2 k(n-k+1)} \leq|M \ddot{o} b(n)|_{k} \leq 2 n+2+k,
$$

where $|\cdot|_{k}$ denotes $S_{k}$-length.

In particular, we have

Corollary 1.2 (Linear growth). For a fixed codimension $k$ :

- If $k$ is even, $\left|M \ddot{o} b^{+}(n)\right|_{k} \asymp n$.

- If $k$ is odd, $|\operatorname{Möb}(n)|_{k} \asymp n$.

Received by the editors August 15, 2010 and, in revised form, April 19, 2011. 2010 Mathematics Subject Classification. Primary 51M10; Secondary 30F40.

The first author was supported in part by PSC-CUNY Grant 627 14-00 40.

(C)2012 American Mathematical Society Reverts to public domain 28 years from publication 
Our main tool in achieving both lower bounds in Theorem 1.1 is a dimension count which yields the fact that the set of elements of the form $\alpha_{1} \ldots \alpha_{m}$, where $\alpha_{i}$ is a $k$-involution and $m<\frac{n(n+1)}{2 k(n-k+1)}$, has measure zero in Möb(n) (see Theorem 4.1 and Corollary 2.2). The upper bounds follow from elementary considerations (see Corollary 2.2).

We next consider the percentage of involution conjugacy classes for which $\left|\mathrm{Möb}^{+}(n)\right|=2$. More precisely, define

$$
\Phi(n)=\frac{\mid\left\{k: \text { the } S_{k} \text {-length of } \mathrm{Möb}^{+}(n) \text { is } 2\right\} \mid}{\mid\{\text { involution conjugacy classes in dimension } n\} \mid} .
$$

Recall that the conjugacy class of an involution is determined by the dimension of its (totally geodesic) fixed point set. Thus a $k$-involution determines a conjugacy class, and the denominator above is $n$. Setting $\left|\mathrm{Möb}^{+}(n)\right|=2$ and using the lower bound in inequality (11), after a straightforward computation to find bounds on $k$ in terms of $n$ we have

Corollary 1.3. $\Phi(n)=O\left(n^{-\frac{1}{2}}\right)$.

Remark 1.4. In the paper 1 the authors show that in each dimension $n$ there exists an involution conjugacy class $S_{k}$ for which $\left|\mathrm{Möb}^{+}(n)\right|=2$. When $n$ is even, $k$ may be taken to be $\frac{n}{2}$, and when $n$ is odd, $k$ can be taken to be $\frac{n+1}{2}$. The results of our paper show that away from the middle codimensions (relative to $n$ ) one cannot expect the length of $\mathrm{Möb}^{+}(n)$ to be small. Factoring of isometries from a geometric viewpoint in hyperbolic 4 -space is also studied in 3 .

Hyperbolic $n$-space is denoted by $\mathbb{H}^{n}$. The group of orientation-preserving isometries of $\mathbb{H}^{n}$ (the Möbius group) is $\mathrm{Möb}^{+}(n)$, and the full group is $\operatorname{Möb}(n)$. An involution is an order-two isometry of $\mathbb{H}^{n}$ and a $k$-involution is an isometry with a fixed point set of codimension $k$. A reflection is an involution with a codimension one fixed point set, and a half-turn is an involution with a codimension two fixed point set. The involution is orientation-reversing if and only if the codimension of the fixed point set is odd. For $k=1,2,3, \ldots, n$, let $S_{k}$ be the set (conjugacy class) of $k$-involutions. For the basics on hyperbolic space and its isometry group we refer to Maskit or Ratcliffe (2, 4]). For standard material on differential topology and Lie groups the reader is referred to [5] and [6].

We will use the well-known facts that the dimension of $O(n)$, as well as $S O(n)$, is $\frac{n(n-1)}{2}$ and the dimension of $\mathrm{Möb}^{+}(n)$, as well as $\operatorname{Möb}(n)$, is $\frac{n(n+1)}{2}$.

The paper is organized as follows. Section 2 contains the proofs that conjugacy classes of involutions generate the Möbius group as well as upper bounds on word length. In section 3, we show that the space of $k$-involutions is a submanifold of $\operatorname{Möb}(n)$ having dimension $k(n-k+1)$. Finally, we prove Theorem 1.1]in section 4 .

\section{2. $k$-INVOLUTIONS IN THE ORTHOGONAL AND MÖBIUS GROUPS}

Throughout this section, we fix an integer $n \geq 2$ and an integer $k=1,2, \ldots, n-1$. The case $k=n$ is excluded since $S_{n} \cap O(n)$ has only one element and hence does not generate the orthogonal group.

This section is devoted to proving 
Theorem 2.1. $S_{k} \cap O(n)$ generates $O(n)$ if $k$ is odd and generates $S O(n)$ when $k$ is even. Furthermore for $g \in O(n)$,

$$
|g| \leq\left\{\begin{array}{ll}
2 n, & \text { if } g \text { is orientation-preserving } \\
2 n-2+k, & \text { if } g \text { is orientation-reversing }
\end{array}\right\},
$$

where $|g|$ is the $S_{k} \cap O(n)$-length of $g$.

Corollary 2.2. $S_{k}$ generates $M o ̈ b(n)$ if $k$ is odd and generates $M \ddot{b} b^{+}(n)$ when $k$ is even. Furthermore for $g \in M o ̈ b(n)$,

$$
|g| \leq\left\{\begin{array}{ll}
2 n+4, & \text { if } g \text { is orientation-preserving } \\
2 n+2+k, & \text { if } g \text { is orientation-reversing }
\end{array}\right\},
$$

where $|g|$ is the $S_{k}$-length of $g$.

Remark 2.3. In both Theorem 2.1 and Corollary 2.2, we note that $k$ is necessarily odd when $g$ is orientation-reversing.

The stabilizer of any point in $\mathbb{H}^{n}$ has a natural identification with $O(n)$. We fix such a copy of $O(n) \subset \operatorname{Möb}(n)$.

Denote the $n \times n$ diagonal matrices with $k$ entries being -1 and with $n-k$ entries being 1 by $\mathcal{D}(n, k)$. Since an involution in $O(n)$ is $O(n)$-conjugate to a diagonal matrix, it is immediate that a $k$-involution in $O(n)$ is conjugate to a diagonal matrix in $\mathcal{D}(n, k)$. There are $\left(\begin{array}{c}n \\ k\end{array}\right)$ such matrices.

Lemma 2.4. Assume $n \geq 2$ and $k=1, \ldots, n-1$.

(1) If $k$ is odd, then any element of $\mathcal{D}(n, 1)$ can be written as the product of $k$ elements of $\mathcal{D}(n, k)$.

(2) Any element of $\mathcal{D}(n, 2)$ can be written as the product of two elements of $\mathcal{D}(n, k)$.

Proof. For ease of notation, we identify the diagonal matrices of size $n$ having \pm 1 entries with the group $\mathbb{Z}_{2}^{n}$. That is, $\bigcup_{k=0}^{n} \mathcal{D}(n, k)=\mathbb{Z}_{2}^{n}$. We write an element of $\mathbb{Z}_{2}^{n}$ as a vector with the obvious component-wise multiplication in $\mathbb{Z}_{2}$.

To prove item (1), consider $A=[-1,1,1, \ldots, 1] \in \mathcal{D}(n, 1)$. It suffices to show that $A$ can be written as the desired product. For $i=1, \ldots, k$, let $C_{i} \in \mathcal{D}(n, k)$ with $j$-th component being

$$
C_{i}^{j}=\left\{\begin{array}{l}
1, \text { if } j=i+1 \text { or } k+2 \leq j \leq n \\
-1, \text { if } 1 \leq j \leq k+1 \text { and } j \neq i+1
\end{array}\right\}
$$

Then $A=\prod_{i=1}^{k} C_{i}$ and we have the desired decomposition of $A$.

To prove item (2), consider $A=[-1,-1,1, \ldots, 1] \in \mathcal{D}(n, 2)$. It suffices to show that $A$ can be written as the desired product. Let $R \in \mathcal{D}(n, k)$ be such that its $j$-th component is

$$
R^{j}=\left\{\begin{array}{l}
1, \text { if } j=1 \text { or } k+2 \leq j \leq n \\
-1, \text { if } 2 \leq j \leq k+1
\end{array}\right\}
$$

and let $S \in \mathcal{D}(n, k)$ have $j$-th entry

$$
S^{j}=\left\{\begin{array}{l}
1, \text { if } j=2 \text { or } k+2 \leq j \leq n \\
-1, \text { otherwise }
\end{array}\right\} .
$$

Then $R S=A$ and we are finished with the proof of item (2). 
Lemma 2.5. Let $a$ and $b$ be reflections in hyperplanes $\alpha$ and $\beta$ in $\mathbb{H}^{n}(n \geq 3)$ and let $g=a b$. Then there exist half-turns $h$ and $k$ such that $g=h k$.

Proof. Consider the upper half-space model of $\mathbb{H}^{n}$. Let $\alpha \cap \hat{\mathbb{R}}^{n-1}=\tilde{\alpha}$ and $\beta \cap \hat{\mathbb{R}}^{n-1}=$ $\tilde{\beta}$. Then $\tilde{\alpha}$ and $\tilde{\beta}$ are $(n-2)$-spheres in $\hat{\mathbb{R}}^{n-1}$. We may assume that neither $\tilde{\alpha}$ nor $\tilde{\beta}$ contains the point at infinity $(\infty)$. Consider the unique circle $\rho$ through $\infty$ and each of the centers of $\tilde{\alpha}$ and $\tilde{\beta}$. It is clear that any $(n-2)$-sphere containing $\rho$ is orthogonal to each of $\tilde{\alpha}$ and $\tilde{\beta}$. Let $\tilde{\gamma}$ be one such $(n-2)$-sphere.

Then, $\tilde{\gamma}=\gamma \cap \hat{\mathbb{R}}^{n-1}$, where $\gamma$ is a hyperplane in $\mathbb{H}^{n}$ which is orthogonal to each of $\alpha$ and $\beta$. Let $c$ denote reflection in $\gamma$. Then $h=a c$ and $k=c b$ are half-turns in $\mathbb{H}^{n}$ such that $h k=(a c)(c b)=a c c b=a b=g$.

Proof of Theorem 2.1. Fix $k=1, \ldots, n-1$. Using the block diagonal form for an element in $g \in S O(n)$, it is easy to see that an element $g \in S O(n)$ can be written as a product $\rho_{1} \ldots \rho_{m}$, where $\rho_{i} \in S_{1} \cap O(n), m$ is even, and $m$ is at most $n$. Now, using Lemma 2.5. we write $g$ as a product of $m$ half-turns. Of course, the halfturns are $O(n)$-conjugate to a diagonal matrix in $\mathcal{D}(n, 2)$ and hence using item (2) of Lemma 2.4, we can write $g$ as the product of at most $2 n$ elements in $S_{k} \cap O(n)$.

If $g \in O(n)-S O(n)$, then $g=\rho_{1} \ldots \rho_{m}$, where $m$ is odd and at most $n$. Note that it must be that $k$ is odd. As above we write $\rho_{1} \ldots \rho_{m-1}$ as the product of at most $2 n-2$ elements in $S_{k} \cap O(n)$. The reflection $\rho_{m}$, using item (1) of Lemma 2.4 can be written as the product of $k$ elements in $S_{k} \cap O(n)$. Thus for such an element $g$, $|g| \leq 2 n-2+k$.

Proof of Corollary 2.2, For $g \in \operatorname{Möb}(n)$, it is well known that $g=\Phi \tau \sigma$, where $\sigma$ and $\tau$ are reflections, and $\Phi$ is an element of $O(n)$. Moreover $g$ is orientationpreserving if and only if $\Phi \in S O(n)$. Using Lemma 2.5, we can replace $\tau \sigma$ by the product of two half-turns which by Lemma 2.4 can be written as the product of 4 elements in $S_{k}$. The corollary now follows from Theorem 2.1.

\section{InVOLUtions AND the SPACE of TOTALLY GEODESIC SUBSPACES OF $\mathbb{H}^{n}$}

Throughout this section, we fix an integer $n \geq 2$ and an integer $k=1,2, \ldots, n-1$.

Lemma 3.1. $S_{k} \subset M o ̈ b(n)$ is a (connected) differentiable submanifold of dimension $k(n-k+1)$.

Proof. Set $G=\operatorname{Möb}(n)$. Fix $\alpha \in S_{k} \subset \operatorname{Möb}(n)$ and denote its fixed point set by $\pi$, an $(n-k)$-dimensional plane. Consider the smooth conjugation action of the Lie group $G$ on itself, namely, $g \cdot f=g f g^{-1}$. Since an orbit of a Lie group action is a submanifold, we have that the $G$-orbit of $\alpha$, that is $S_{k}$, is a submanifold of $\operatorname{Möb}(n)$. Furthermore, the map from $G$ to $G$, given by $g \mapsto g \alpha g^{-1}$, induces a one-to-one smooth map from $G / K$ onto $S_{k}$, where $K=\operatorname{Stab}_{G}(\alpha)$. (Note that $K$ is a closed subgroup of $G$.) Next observe that $\operatorname{Stab}_{G}(\alpha)=\operatorname{Stab}_{G}(\pi)$ and consider the map

$$
\Phi: \operatorname{Stab}_{G}(\pi) \rightarrow \operatorname{Möb}(n-k),
$$

given by $\left.g \mapsto g\right|_{\pi}$. This is a surjective map with kernel being isomorphic to $O(k) \leq$ $\operatorname{Stab}_{G}(\pi)$. Hence, $\operatorname{Stab}_{G}(\pi) / O(k)$ is isomorphic to $\operatorname{Möb}(n-k)$, and thus

$$
\operatorname{dim}(K)=\operatorname{dim}\left(\operatorname{Stab}_{G}(\alpha)\right)=\operatorname{dim}(\operatorname{Möb}(n-k))+\operatorname{dim}(O(k)) .
$$

Thus we have

$$
\operatorname{dim}\left(S_{k}\right)=\operatorname{dim}(G)-\operatorname{dim}(K)=\operatorname{dim}(G)-\operatorname{dim}(\operatorname{Möb}(n-k))-\operatorname{dim}(O(k)) .
$$


Now plugging in the various quantities and simplifying yield the dimension of $S_{k}$ to be $k(n-k+1)$.

For $k=1, \ldots, n-1$, let $\mathcal{G}_{k}$ denote the space of $k$-planes (that is, $k$-dimensional totally geodesic subspaces) in $\mathbb{H}^{n}$. The boundary (at infinity) of a $k$-plane is a round $(k-1)$-sphere. The space of $(k-1)$-spheres with the Gromov-Hausdorff topology induces a natural topology on $\mathcal{G}_{k}$.

Corollary 3.2. $\mathcal{G}_{k}$ is a differentiable manifold of dimension $(n-k)(k+1)$.

Proof. Consider the map $\mathcal{G}_{k} \rightarrow \mathcal{S}_{n-k}$ given by taking the $k$-plane $\pi$ to the $(n-k)$ involution with fixed point set $\pi$. As can be checked by the reader, this map is a homeomorphism. Pulling back the differentiable structure from $\mathbb{S}_{n-k}, \mathcal{G}_{k}$ becomes a differentiable manifold whose dimension by Lemma 3.1 is $(n-k)(k+1)$.

\section{Bounds for the $S_{k}$-Length of the Möbius group}

Given a subset $J \subseteq\{1, \ldots, n-1\}$, let $S$ be the generating set $S=\bigcup_{k \in J} S_{k}$, and set $M=M(S)=\max _{c \in J}\left\{\operatorname{dim}\left(S_{k}\right)\right\}=\max _{k \in J}\{k(n-k+1)\}$.

Theorem 4.1. Except for a set of measure zero, no element of Möb(n) can be written as a product $\alpha_{1} \ldots \alpha_{m}$, where $\alpha_{i} \in S$, and $m<\frac{n(n+1)}{2 M}$.

Proof. Consider the manifold which is the $m$-fold product of the Möbius group. Given a sequence $\left\{k_{1}, \ldots, k_{m}\right\}$ of $m$-elements from $J$ (repetition is allowed), consider the mapping $\Psi: S_{k_{1}} \times \ldots \times S_{k_{m}} \rightarrow \operatorname{Möb}(n)$ which assigns the $m$-tuple of ordered $k_{i}$ involutions $\left(\alpha_{1}, \ldots, \alpha_{m}\right)$ to the product $\alpha_{1} \ldots \alpha_{m}$. This is a smooth mapping between manifolds. The dimension of $S_{k_{1}} \times \ldots \times S_{k_{m}}$ is bounded from above by $m M$, which is by assumption less than the dimension of $\operatorname{Möb}(n)=\frac{n(n+1)}{2}$. Hence $\Psi$ is a smooth mapping from a manifold of lower dimension to one of higher dimension. It is a standard fact that the image of a smooth map from a manifold of lower dimension to one of higher dimension has measure zero.

Finally, there are a finite number of (namely, $\sum_{i=1}^{\left[\frac{n(n+1)}{2 M}\right]}|J|^{i}$ ) sequences from $J$ of length less than or equal to $\frac{n(n+1)}{2 M}$, and hence a finite number of maps $\Psi$ above. Thus the set of elements in $\operatorname{Möb}(n)$ that are in the images of such maps $\Psi$ is the finite union of sets of measure zero, hence has measure zero. This precisely says that the set of elements of $\operatorname{Möb}(n)$ that can be written as a product of at most $m$ elements from $S$ has measure zero.

Theorem 1.1. Let $n \geq 2$ and $k=1,2, \ldots, n-1$.

- If $k$ is even, $S_{k}$ generates $\mathrm{Möb}^{+}(n)$ and satisfies

$$
\frac{n(n+1)}{2 k(n-k+1)} \leq\left|M \ddot{o} b^{+}(n)\right|_{k} \leq 2 n+4 .
$$

- If $k$ is odd, $S_{k}$ generates $M o ̈ b(n)$ and satisfies

$$
\frac{n(n+1)}{2 k(n-k+1)} \leq|M \ddot{o b}(n)|_{k} \leq 2 n+2+k .
$$

Proof. The upper bound in either the even or odd case follows from Corollary 2.2 , For the lower bound, if all $k_{i}=k$, then $M=k(n-k+1)$. Now if $|g|<\frac{n(n+1)}{2 k(n-k+1)}$, for all $g \in \operatorname{Möb}(n)$, then Theorem 4.1 is contradicted. 


\section{ACKNOWLEDGEMENTS}

The second author is indebted to his advisor, Jane Gilman, and would like to thank Rutgers-Newark for its support. It is a pleasure to thank Mark Feighn, Lee Mosher and Jacob Sturm for helpful conversations and encouragement.

\section{REFERENCES}

[1] A. Basmajian and B. Maskit, Space form isometries as commutators and products of involutions, Trans. Amer. Math. Soc., to appear.

[2] B. Maskit, Kleinian groups, Grundlehren der Mathematischen Wissenschaften [Fundamental Principles of Mathematical Sciences], 287. Springer-Verlag, Berlin, 1988. MR959135 (90a:30132)

[3] K.M. Puri, Factorization of isometries of hyperbolic 4-space and a discreteness condition. Thesis, Rutgers University, 2009. MR2713266

[4] J. G. Ratcliffe, Foundations of hyperbolic manifolds. Second edition, Graduate Texts in Mathematics, 149. Springer, New York, 2006. MR2249478(2007d:57029)

[5] M. Spivak, A comprehensive introduction to differential geometry, Vol. I. Second edition. Publish or Perish, Inc., Wilmington, Del., 1979. MR.532830 (82g:53003a)

[6] J. Wolf, Spaces of Constant Curvature. Fifth edition, Publish or Perish, Houston, TX, 1984. MR0928600(88k:53002)

Department of Mathematics, Graduate Center and Hunter College, CUny, New York, New YoRK 10016

E-mail address: abasmajian@gc.cuny.edu

Department of Mathematics, Queensborough Community College, Cuny, Bayside, NEW YORK 11364

E-mail address: kpuri@qcc.cuny.edu 